Mittheilung ans dem ohemischen Laboratorium der Königl, Bergakademie zu Freiberg (Sachsen).

Ueber die Einwirkung von hydroschwefligsaurem Natrium auf Metallsalze;

von O. Brunck.

[Zweite Mittheilung.]

(Eingelanfen am 17. Angust 1904.)

Nachdem ich im vorigen Jahre in dieser 'Zeitschrift ${ }^{1}$ ) uber die Einwirkung von hydroschwefligsaurem Natrium auf die Salze von Kupfer, Silber, Gold, Zink, Cadmium und Quecksilber berichtet habe, will ich jetzt das Verhalten dieses Reagenzes auf die Salze der ubrigen wichtigeren Metalle beschreiben. In den Rahmen der Untersuchung sind auch die Verbindungen einiger Halbmetalle und Motalloïde einbezogen worden.

\title{
7. Aluminium.
}

Aluminium wird aus den Lösungen seiner Salze, sofern freie Säure nicht zugegen ist, durch Natriumbydrosulfit beim Kochen als Hydroxyd gefällt, dem etwas Schwefel beigemengt ist. Doch ist die Fällung gerade wie beim Natriumthiosulfat, dem das Hydrosulfit bezüglich seines Verhaltens zu Metallsalzen in mancher Hinsicht ăhnelt, nie ganz vollständig. Vielleicht wird dies verursacht durch die Bildung eines Sulfits, das beim Kochen nicht völlig zersetzt wird.

\section{Indium.}

Neutrale Indiumlösungen werden bei gewöhnlicher Temperatur durch Natriumbydrosulfit nicht gefällt; erhitzt man zum Sieden, so scheidet sich das Indium als Sulfid in Form eines strohgelben Niederschlages aus. Vermuthlich bildet sich leicht

1) Diese Annalen 397, 240 (1903). 
lösliches Indiumbydrosulfit, das beim Kochen unter Abscheidung von Indiumsulfid zersetzt wird. Bei Gegenwart freier Säure entsteht kein Niederschlag. Aber auch in neutraler Lösung ist die Fällung unvollständig in Folge der lösenden Wirkung der bei der Reaction entstehenden freien schwefligen Säure.

\section{Thallizm.}

Versetzt man die neutrale Lösung eines Thalliumsalzes mit Natriumbydrosulfit, so entsteht schon in der Kälte langsam ein rothbrauner, allmählich violett und dann schwarz werdeuder Niederschlag von Thalliumsulfür. Die Fällung bleibt auch beim Erhitzen unvollständig aus dem gleichen Grunde wie beim Indium. Säuert man die Lösung von vornherein ganz schwach an, so entsteht der rothbraune Niederschlag sofort. Ist mehr Säure zugegen, so tritt uberhaupt keine Fällung ein.

\section{Titan.}

Dass neutrale oder schwach saure Titanlösungen durch hydroschweflige Säure intensiv rothviolett gefärbt werden, ist längst bekannt. R. Fresenius ${ }^{2}$ ) empfahl diese Reaction zum Nachweise von Titan. Mit Hülfe derselben lassen sich noch $0,02 \mathrm{mg}$ Titandioxyd in $1 \mathrm{ccm}$ erkennen. Sie ist daher fast ebenso scharf wie die mit Wasserstoffsuperoxyd. Frosenius nahm a11, dass die Reaction auf einem Reductionsvorgange beruhe; damit schien aber die ron mir beobachtete Thatsache im Widerspruche zu stehen, dass bei Zusatz von Ammoniak zu der rothen Lösung ohne Gasentwickelung ein Niederschlag von Titansäurehydrat entsteht, unter gleichzeitiger Entfärbung der Flüssigkeit, wäbrend in Lösungen des dreiwerthigen Titans durch Ammoniak dunkelrothe bis schwarzviolette Niederschläge erhalten werden. Inzwischen fand nun Edmund Knecht ${ }^{3}$ ), dass schweflige Säure durch Titantrichlorid zu hydroschwefliger Säure reducirt wird und dass man auf diese Weise letztere leicht in Form

2) Zeitschr. f. analyt. Chem. 24, 410 (1885).

s) Ber. d. deutsch. chem. G'es. 36, 166 (1903). 
ihrer Salze gewinnen kann, wenn man die mit Titantrichlorid versetzte Lösung von schwefliger Säure durch Alkali neutralisirt. Wir haben es also hier mit einer umkehrbaren Reaction zu thun:

$$
2 \mathrm{TiCl}_{4}+\mathrm{Na}_{2} \mathrm{~S}_{2} \mathrm{O}_{4} \longleftrightarrow 2 \mathrm{TiCl}_{3}+2 \mathrm{NaCl}+2 \mathrm{SO}_{3} .
$$

In saurer Lösung bei einem Ueberschusse von hydroschwefliger Säure vollzieht sich die Reaction im Sinne von links nach rechts, in alkalischer von rechts nach links. In Uebereinstimmung hiermit steht die Thatsache, dass alkalische Titanatlösungen mit Natriumbydrosulfit keine Rothfärbung ergeben.

\section{Germanium.}

Germanium wird weder aus neutraler noch aus saurer Lösung durch Natriumhydrosulfit gefällt. Selbst in sehr stark saurer Lösung entsteht nicht, wie man erwarten könnte, ein Niederscblag von Germaniumsulfid, sondern lediglich eine starke Schwefelausscheidung.

\section{Zirkon.}

Gegen Zirkon verhält sich Natriumhydrosulfit wie gegen Aluminium. Fügt man das Reagens zu der Lösung eines neutralen Zirkonsalzes und kocht, so entsteht ein weisser Niederschlag von Zirkonsäurehydrat, gemengt mit Schwefel. Bei Gegenwart freier Süure tritt keine Fällung ein.

\section{Zinn.}

Fügt man zu einer ziemlich concentrirten, schwach salzsauren Lösung von Zinnchlorür eine zehnprocentige Lösung von Natriumbydrosulfit, so entsteht augenblicklich ein weisser, voluminöser Niederschlag, der rasch krystallin wird und sich im Ueberschusse des Fällungsmittels mit Leichtigkeit wieder löst. Zur näheren Untersuchung desselben wurde die Zinnchlorürlösung tropfenweise mit Hydrosulfit versetzt, so lange in der über dem sich rasch absetzenden Niederschlage stehenden Flüssigkeit noch eine Fällung eintrat. Der Niederschlag wurde dann möglichst rasch mit wenig kaltem Wasser ausgewaschen, 
wobei er sich stets gelblich färbte, und dann über Schwefelsäure im Vacuum getrocknet bis zur Gewichtsconstanz. Man erhielt so ein lockeres, gelbliches, manchmal auch röthliches Pulver, das stets noch Wasser enthielt, aber neben Zinn nur Spuren von Natrium. In Wasser suspendirt reducirt die Verbindung schon bei gewöhnlicher Temperatur Kupfersalze zu metallischem Kupfer. Wir haben es also offenbar mit einem Zinnsalze der hydroschwefligen Säure zu thun, das mit überschüssigem Natriumhydrosulfit ein leicht lösliches Doppelsalz bildet. Doch gelang es nicht, eine Verbindung von constanter Zusammensetzung zu erhalten. Die Analyse des in verschiedenen Operationen dargestellten Productes ergab ganz wechselnde Gehalte an Zinn und Schwefel. Das Verbältniss beider Elemente entsprach niemals einer bestimmten Formel. Wahrscheinlich erleidet das anfangs gebildete Zinnhydrosulfit schon während des Versuches, es zu isoliren, eine theilweise Zersetzung und ist schliesslich mehr oder minder stark durch die Zersetzungsproducte verunreinigt.

Versetzt man eine verdünnte Zinnchlorürlösung, die etwas mehr freie Säure enthält, mit dem Reagens, so trubt sich die anfangs klare Flüssigkeit nach kurzer Zeit und scheidet einen braunen Niederschlag von Zinnsulfür aus. In der Wärme entsteht derselbe augenblicklich. Zinnsulfür, welches denınach das Endproduct der Zersetzung des primär gebildeten Zinnlydrosulfits ist, dürfte aber in dem oben beschriebenen Reactionsproducte wegen dessen schwacher Färbung kaum in grösserer Menge enthalten sein. Wahrscheinlich bilden sich complicirte Zwischenglieder.

Aus der Lösung von Zinnoxydsalzen fällt Natriumhydrosulfit in der Kälte langsam, rasch beim Erwärmen gelbes Zinnsulfid. Die Fällung ist vollständig.

\section{Blei.}

Da die Natriumhydrosulfitlösung auch in frisch bereitetem Zustande stets etwas Sulfit und Thiosulfat enthielt, diese aber mit Blei unter Bildung schwer löslicher Salze reagiren, wurde 
das Reagens zuerst durch tropfenweisen Zusatz von Baryumchlorid von diesen Verunreinigungen befreit. Die abfiltrirte Lösung erzeugt in neutralen Bleisalzlösungen sofort einen gelblich-weissen Niederschlag, der rasch roth, braun und schliesslich schwarz wird. Vermuthlich entsteht primär ein schwerlösliches, sehr unbeständiges Bleisalz der hydroschwefligen Săure, das rasch in Bleisulfid übergeht. Dieser Uebergang vollzieht sich um so rascher, je grösser der Ueberschuss des Fällungsmittels ist. Tropft man daher umgekehrt das Bleisalz in eine nicht zu verdünnte Lösung von Natriumhydrosulfit, so entsteht sofort ein schwarzer Niederschlag von Schwefelblei. In einer freie Salzsäure enthaltenden Bleilösung ruft das Reagens gleich einen rothen Niederschlag von Bleichlorosulfid hervor, der durch einen Ueberschuss rasch in schwarzes Bleisulfid umgewandelt wird. Die Fällung des Bleis ist eine vollständige.

\section{Arsen.}

Den Sauerstoffverbindungen des Arsens gegenüber zeigt sich Natriumbydrosulfit als ein höchst energisches Reductionsmittel, indem es dieselben zu elementarem Arsen reducirt. Fügt man das Reagens zu einer mit Salzsäure schwach angesäuerten Lösung von arseniger Säure, so entsteht sofort ein rothbrauner, amorpher, beim Umrühren sich zusammenballender Niederschlag, der sich leicht filtriren und auswaschen lässt. Bei Abwesenheit freier Säure entsteht der Niederschlag erst nach einiger Zeit, während eine mit Natriumbydrocarbonat alkalisch gemachte Lösung von arseniger Säure uberhaupt nicht verändert wird.

Im Vacuum uber Schwefelsäure getrocknet stellt der Niederschlag ein chocoladebraunes Pulver dar, das unter dem Pistille Bronzeglanz annimmt. An der Luft erhitzt verbrennt es mit fahlblauer Flamme zu Arsentrioxyd; mit rauchender Salpetersäure befeuchtet oxydirt es sich unter glänzender Lichterscheinung. Der frisch gefällte Niederschlag löst sich leicht in warmer, verdunnter Salpetersäure, ist aber unlöslich in Ammoniak und farblosem Schwefelammonium. Erwärmt man 
ihn mit gelbem Schwefelammonium, so wird dieses entfärbt, während ein Theil des Niederschlages als Sulfosalz in Lösung geht. Ammoniakalisches Wasserstoffsuperoxyd löst ihn leicht zu Ammoniumarseniat.

Anfänglich wurde vermuthet, es liege fester Arsenwasserstoff vor, der von Davy, Gay-Lussac und Thénard, Magnus und Janowski als braunes Pulver beschrieben wird, das beim Erhitzen in einer sauerstofffreien Atmosphäre gasförmigen Wasserstoff abspaltet. Eine Probe der getrockneten Substanz wurde in einer schwer schmelzbaren Glasröbre, die mit einer Gasbürette verbunden war und aus der man vorher alle Luft durch Kohlensäure verdrängt hatte, erhitzt bis zum beginnenden Schmelzen. Das braune Pulver ging dabei in graues, krystallines Arsen uber, ohne dass jedoch die geringste Gasentwickelung bemerkbar war. Demuach ist das Product der Einwirkung von hydroschwefliger Säure auf arsenige Säure amorphes, braunes Arsen.

Um amorphes Arsen auf diese Weiso in möglichst reinem Zustande zu erhalten, ist ein Säureüberschuss thunlichst zu vermeiden. Am besten verfährt man so, dass man eine kalt gesättigte wässrige Lösung von arseniger Säure mit überschüssigem Natriumhydrosulfit versetzt und dann tropfenweise Salzsäure unter Umrühren zufügt, so lange noch cin brauner Niederschlag entsteht. Aber auch das so erhaltene Arsen enthält stets noch sehr geringe Mengen von Schwefel. Derselbe ist weder als freier Schwefel vorhanden, da der Niederschlag beim Erhitzen mit Natriumsulfitlösung an diese keinen Schwefel abgiebt, noch in Form von Arsentrisulfid; denn Ammoniak vermag kein Arsen herauszulösen. Kocht man jedoch den Niederschlag mit Kalilauge und säuert die abfiltrirte Lösung an, so entsteht ein geringer Niederschlag von gelbem Arsensultid. Dies Verhalten deutet auf die Anwesenheit von Arsendisulfid hin, welches in Ammoniak nicbt löslich ist, aber beim Kochen mit Kalilauge zersetzt wird unter Abscheidung von Arsen und Bildung von löslichem Kaliumsulfarsenit. 
Nach W. F. Cooke ${ }^{4}$ ) wird amorphes Arsen von Wasser etwas gelöst, jedoch weit weniger als krystallines. Thatsächlich geht beim Auswaschen des Niederschlags mit Wasser stets etwas Arsen in Lösung. Durch Zusatz von schwefliger Säure zum Wascbwasser lässt sich dies jedoch vermeiden.

Fällt man Arsen aus stark saurer Lösung durch Natriumhydrosulfit, so sind dem Niederschlage ausser freiem Schwefel nicht unbeträchtliche Mengen von Arsentrisulfid beigemengt, zumal wenn die Fällung in der Wärme erfolgt. In diesem Falle enthält dann das Filtrat stets etwas Arsen, gleichgültig ob man das Auswaschen mit oder ohne Zusatz von schwefliger Säure bewirkt. Dieser Umstand beschränkt die Anwendung des Natriumhydrosulfits zur Fällung des Arsens bei der chemischen Analyse, wo die Anwesenheit erheblicher Mengen ron freier Säure meist nicht zu vermeiden ist, auf solche Fälle, wo es sich darum handelt, bedeutende Mengen von Arsen bis auf einen unbedeutenden Rest rasch aus einer Lösung zu entfernen. Die Thatsache, dass die Fällung des Arsens auch aus einer Lösung von Arsensäure erfolgt und dass der Niederschlag sehr dicht ist, so dass selbst grosse Mengen auf einem verhältnissmässig kleinen Filter gesammelt und leicht ausgewaschen werden könnell, lassen das Natriumhydrosulfit hierzu als höchst geeignet erscheinen.

\section{Antimon.}

Antimon verbält sich in den Lösungen seiner Salze gegenuber Natriumhydrosulfit ganz ähnlich wie Kupfer. Bei unzureichenden Mengen des Reagens wird metallisches Antimon abgeschieden, bei einem Ueberschusse desselben Sulfid. Nur erfolgt die Reduction schwieriger als beim Kupfer, erst in der Wärme, und die Neigung zur Sulfidbildung ist grösser.

Fügt man zu einer schwach sauren Lösung von Antimonchlorid uberschüssiges Natriumbydrosulfid, so entsteht in der

4) Proc. chem. Soc. 19, 243 (1902). 
Kälte längsam, beim Erwärmen rasch ein orangerother Niederschlag von Antimontrisulfid, gemengt mit Schwefel. Tropft mau jedoch langsam das Reagens zu der heissen Antimonlösung, so scheidet sich metallisches Antimon als schwarzer, dichter Niederschlag ab. Jedoch ist es nicht möglich, alles Antimon als Metall zu fällen, da die letzten Antheile erst bei längerem Erwärmen mit einem Ueberschusse des Reagenzes abgeschieden werden, wodurch die Bildung von Sulfid bedingt ist.

Aus einer neutralen oder alkalischen Lösung von Kaliumantimoniat scheidet Natriumhydrosulfid in der Wärme metallisches Antimon ab, zum Theil als an dem Glase festhaftenden spiegelnden Belag, gleichgültig, ob das Reagens in unzureichender Menge oder im Ueberschusse zugefügt wird. Eine angesäuerte Lösung von Kaliumantimoniat verhält sich wie eine Losung vou Antimontrichlorid. Bei Ueberschuss von Hydrosulfit fällt rotbes Sulfid, im entgegengesetzten Falle schwarzes Antimon.

In allen Fällen konnte im Filtrate noch Antimon nachgewiesen werden. Zur quantitativen Abscheidung des Antimons ist die Reaction daher nicht geeignet.

\section{Wismuth.}

Das Wismuth ähnelt in seinem Verhalten zu hydroschwefliger Säure dem Kupfer noch mehr als das Antimon. Die Reduction zu Metall erfolgt schon bei gewöhnlicher Temperatur. Giebt man zu einer schwach sauren Lösung von Wismuthchlorid Natriumbydrosulfit, so entsteht ein röthlicher Niederschlag, vielleicht Wismuthhydroxylul, der rasch schwarz wird unter Uebergang in metallisches Wismuth. Derselve setzt sich auch beim Cmrübren nur langsam zu Boden; noch lange erscheint die über dem Niederschlage stehende Flüssigkeit von fein vertheiltem Metall bläulich gefärbt. Erwärmt man den ausgewaschenen Niederschlag mit Salzsäure, so wird er grau und dicht und nimmt beim Drücken mit einem Glasstabe Metallglanz an. Hierbei gehen sebr geringe Mengen von Wismuth in Lösung, die als Sulfid rorbanden waren. 
Fügt man umgekehrt die Wismuthlösung zu überschüssigem Hydrosulfit, so entsteht zuerst ebenfalls der röthliche Niederschlag, der aber rasch in schwarzbraunes Wismuthsulfid umgewandelt wird. Beim Erwärmen mit Salzsüure geht der Niederschlag unter Schwefelwasserstoffentwickelung vollständig in Lösung bis auf etwas Schwefel, der durch Spuren von metallischem Wismuth dunkel gefärbt ist.

Schliesst man freie Săure vollständig aus, indem man das Reagens $\mathrm{zu}$ in Wasser suspendirtem Wismuthhydroxyd giebt, so wird dies, auch wenn jenes im Ueberschusse vorhanden ist, langsam zu Metall reducirt.

Ileintz ${ }^{5}$ ) giebt an, dass das durch Zink gefällte Wismuth sich schon bei $200-220^{\circ}$ beträchtlich oxydire. Das durch Fällen mit Natriumhydrosulfit erhaltene äusserst feine schwarze Metallpulver oxydirt sich aber schon bei $100^{\circ}$ ganz erbeblich. $1 \mathrm{~g}$ des trocknen Pulvers nahm in drei Stunden $1,5 \mathrm{pC}$. seines Gewichts an Sauerstoff auf. Erhitzt man das Pulver bei Luftzutritt stärker, so entzündet sich dasselbe noch weit unter Gluhbitze and verglimmt vollständig zu Wismutboxyd.

Die Fällung des Wismuths ist vollständig, auch in salpetersaurer Lösung, wenn nicht mehr freie Säure vorhanden ist als nöthig ist, um beim Verdünen die Bildung basischen Nitrates hintan zu halten. Da aber auch dieses beim Behandeln mit Natriumbydrosulfit zu Metall reducirt wird, so ist es gleichgultig, ob beim Verdünnen mit Wasser die Wismuthlösung sich trubt oder nicht. Behufs quantitativer Bestimmung kann der getrocknete Niederschlag nicht ohne Weiteres durch Gluhen in wägbares Oxyd ubergefübrt werden. Da er stets etwas. Wismuthsulfid enthält, wird das Oxyd sulfathaltig und dies wird selbst durch starkes Gluhen nicht vollständig zerlegt. Es ist daher erforderlich, den ausgewaschenen Niederschlag wieder in Salpetersäure zu lösen und das Wismuth mit Ammoniumcarbonat zu fällen oder elektrolytisch abzuscheiden. Gegenuber

5) Poggendorf's Amalen 63, 58 (1844). 
dem Schwefelwasserstoff bietet daher das Natriumhydrosulfit in analytischer Hinsicht keine besonderen Vorzuge, ausser bei der Trennung von Zink. Diese kann bekanntlich durch einmalige Fällung mit Schwefelwasserstoff nicht erreicht werden, da sie wegen der Löslichkeit des Schwefelwismuths in Säuren nur aus schwach saurer Lösung erfolgen darf, wobei der Niederschlag dann stets durch Zink verunreinigt ist. Das durch Natriumhydrosulfit gefälte Wismuth ist aber auch bei Gegenwart beträchtlicher Mengen von Zink völlig frei von diesem Metalle, selbst wenn die Fällung nur aus schwach saurer Lösung erfolgt, wie durch eine Reihe von Versuchen dargethan wurde.

$\left.\begin{array}{l}\text { 1) Ange- }\{0,1624 \mathrm{~g} \text { Wismuth } \\ \text { wandt: }\{0,20 \mathrm{~g} \mathrm{Zink}\end{array}\right\}$ Gefunden $0,1807 \mathrm{~g} \mathrm{Bi}_{2} \mathrm{O}_{3}=0,1620 \mathrm{~g}$ Wismuth.

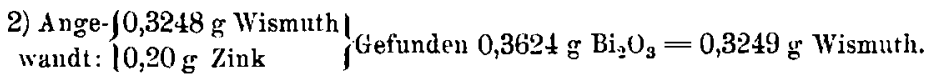

\section{Chrom.}

Chromoxydsalze werden durch Natriumbydrosulfit nicht verändert. Zwar färbt sich die Lösung eines solchen bei $\mathrm{Zu}$ satz des Reagenzes braungelb in Folge der Bildung freier hydroschwefliger Säure; doch zersetzt sich diese beim Stehen unter Schwefelabscheidung, rascher in der Wärme, und die Flüssigkeit nimmt wieder ibre ursprüngliche grüe Farbe an. Auch die violette Lösung des Chromoxyds in Ammoniak wird durch Natriumhydrosulfit nicht verändert. Eine Reduction von Chromoxyd zu Chromoxydul konnte in keinem Falle beobachtet werden.

Chromsäure wie auch Chromate werden durch Natriumbydrosulfit schon bei gewöhnlicher Temperatur augenblicklich zu Chromoxyd reducirt. Die Reduction findet auch bei Gegenwart von Kalilauge statt unter Bildung der grinen Pseudolösung; in der Wärme wird natürlich alles Chrom als Hydroxyd gefällt.

\section{Molybdän.}

Bei der Einwirkung von Natriumhydrosulfit auf Molybdänsäure stand die Bildung der niedrigeren blauen Oxyde zu er- 
warten, wie sie durch andere Keductionsmittel erhalten werden. Doch waltet hier die Neigung zur Sulfidbildung vor. Fügt man das Reagens zu einer schwach angesäuerten Lösung von Ammoniummolybdat, so färbt sich dieselbe sofort rothbraun und scheidet einen braunen Niederschlag von Molybdänsulfid aus. Fine neutrale Lösung von Ammoniummolybdat wird zuerst dunkelgrtin und dann braunroth gefärbt unter Bildung von Ammoniumsulfomolybdat. Beim Ansăuern der Lösung fällt sofort braunes Molybdänsulfid aus.

\section{Wolfram.}

Wolframsäure wird im Gegensatze zur Molybdänsäure durch Natriumhydrosulfit reducirt. Die schwach angesäuerte Lösung von Ammoniumwolframat wird durch das Reagens sofort grü und dann rasch tief blau gefärbt. Bei längerem Stehen tribt sich die blaue Flüssigkeit milchig durch Abscheidung von Schwefel. Eine neutrale Lösung von Ammoniumwolframat wird durch Natriumhydrosulfit nicht verändert. Nach einiger Zeit jedoch tritt eine schwache grunliche Färbung auf.

\section{Uran.}

Natriumbydrosulfit wurde von V. Ko hls $\mathrm{ch} \ddot{\mathrm{u}} \mathrm{tt}$ er und H. Rossib) beuützt zur Reduction von Lranylverbindungen zu Uranosalzen. Diese beiden Forscher geben an, dass in der gelben Lösung der ersteren Natriumbydrosulfit sofort einen braunen Niederschlag hervorrufe, der dann weissgrün wird und sich nach kurzem Erwärmen in Salzsäure mit gruner Farbe zu Uranochlorid löst. Diese Angaben lsann ich bestatigen. Fugt man zur gelben Lösung von Uranylnitrat oder Uranylacetat Natriumbydrosulfit, so erhält man einen rothbraunen Niederschlag, der rasch graugrun wird (ist viel Schwefel beigemengt, so erscheint er heller) und sich im Ueberschusse des Fällungsmittels mit grüner Farbe unter Bildung von Uranosulfit löst. Kocht man diese Lösung, so scheidet sich ein graugrüner

6) Ber. d. deutsch. chem. Ges. 34, 1472 (1901). 
Niederschlag von basischem Uranosulfit aus. Durch schweflige Säure werden bekanntlich Uranylsalze auch in der Wärme nicht reducirt.

\section{Mangan.}

Manganosalze werden durch Natriumhydrosulfit scheinbar nicht verändert, auch nicht in der Wärme. Vielleicht bildet sich ein sehr leicht lösliches Mangansalz der bydroschwefligen Säure, das nur lösliche Zersetzungsproducte liefert. Auch auf Zusatz einer gesättigten Lösung des Reagenzes zu einer kaltgesättigten Lösung von Manganchlorür bleibt diese völlig klar.

Permanganatlösungen werden in saurer wie in neutraler Lösung augenblicklich zu Manganosalz reducirt. Bei Gegenwart von freiem Alkali findet Abscheidung von Superoxyd statt. Die hydroschweflige Säure weicht demnach in ihrem Verhalten gegen Manganverbindungen nicht wesentlich von der schwefligen Säure $a b$.

\section{Eisen.}

Ferrisalze werden durch Natriumhydrosulfit augenblicklich zu Ferrosalzen reducirt. Diese werden in neutraler Lösung durch das Reagens nicht gefällt. Erwärmt man aber die Lösung, so scheidet sich schwarzes Schwefeleisen ab, bei vorsichtigem Erwärmen zum Theil als spiegelnder, am Glase festhaftender Belag. Eine vollständige Fällung lässt sich jedoch in Folge der lösenden Wirkung der bei der Reaction sich bildenden freien schwefligen Säure nicht erzielen. Enthält die Lösung ron vornherein freie Säure, so entsteht natürlich kein Niederschlag.

Natriumthiosulfat soll nach $\mathrm{Gibbs}^{7}$ ) neutrale Ferrosalzlösungen bei fortgesetztem Kochen, ja sogar beim Eindampfen nicht verändern. Versetzt man aber eine solche mit einer nicht $\mathrm{zu}$ verdünnten Lösung von Thiosulfat und erwärmt, so trubt sich die Flüssigkeit nach einiger Zeit und scheidet

7) Zeitschr. f. analyt. Chem. 3, 387 (1864). 
schwarzes Schwefeleisen ab, wenn auch nicht annähernd so viel und so rasch wie mit Hydrosulfit.

\section{Nickel.}

Versetzt man die wässrige Lösung eines Nickelsalzes mit Natriumhydrosulfit, so färbt sich dieselbe gelbbraun, bleibt aber anfangs völlig klar. Allmählich bedecken sich jedoch die Wände des Gefässes mit einem metallisch glänzenden Spiegel von Nickelsulfür. Erwärmt man die Flüssigkeit, so entsteht sofort ein schwarzer Niederschlag von Schwefelnickel. Rascher vollzieht sich die Abscheidung desselben aus einer ammoniakalischen Nickellösung. Quantitativ kann Nickel gefällt werden, wenn man das Reagens in kleinen Portionen unter fortgesetztem Erhitzen zu der neutralen, ammoniakalischen oder essigsauren Lösung zugiebt. Dass aus mineralsaurer Lösung Nickel durch Natriumhydrosulfit nicht gefällt wird, wurde bereits beim Kupfer erwähnt. Ich habe dort auch einige Belege gegeben für die quantitative Trennung der beiden Metalle durch Natriumhydrosulfit. Für gewöhnlich wird man ja bei der Analyse die Trennung dieser Metalle durch Schwefelwasserstoff bewirken oder auf dem Wege der Elektrolyse. In gewissen Fällen gewinnt jedoch die Trennung durch Natriumhydrosulfit praktische Bedeutung, z. B. bei der gleichzeitigen Anwesenheit von Zink; ferner wenn es sich darum handelt, sehr grosse Mengen Kupfer von wenig Nickel zu trennen oder kleine Kupfermengen aus grösseren Volumina Nickellaugen zu entfernen.

Herr Hütteningenieur G. Puchert hat im hiesigen Laboratorium eine Reihe von Versuchen ausgeführt, deren Resultat nachstehend mitgetheilt sei.

Kupfer wurde angewandt in Form einer lösung von reinstem Kupfervitriol, deren Gehalt durch Elektrolyse auf das Genaueste ermittelt war. $1 \mathrm{ccm}$ derselben enthielt $0,005128 \mathrm{~g}$ Kupfer.

Nickel wurde in Fällen, wo es nicht direct bestimmt wurde, als zehnprocentige Lösung von Nickelvitriol angewandt; 
andernfalls als sehr verdünnte Lösung von Nickelsulfat, deren Gehalt ebenfalls durch Elektrolyse festgestellt war. $1 \mathrm{ccm}$ enthielt $0,004622 \mathrm{~g}$ Nickel.

1) Ange- 10,1282 $\mathrm{g}$ Kupfer / Gefunden: $0,1279 \mathrm{~g}$ Kupter, 0,0694 $\mathrm{g}$ Nickel. wandt: $\{0,0693 \mathrm{~g}$ Nickel $\}$

$\begin{array}{lllll}0,1280 \mathrm{~g} & " & 0,0693 \mathrm{~g} & " \\ \mathbf{0 , 1 2 7 9} \mathrm{g} & " & \mathbf{0 , 0 6 9 6} \mathrm{g} & " \\ 0,1280 \mathrm{~g} & " & 0,0694 \mathrm{~g} & " \\ \mathbf{0 , 1 2 7 9} \mathrm{g} & " & 0,0695 \mathrm{~g} & "\end{array}$

2) Angewandt: 10,0256 $\mathrm{g}$ Kupfer( Gefunden: 0,0255 $\mathrm{g}$ Kupfer.

$\begin{array}{rl}\mid 10 \mathrm{~g} \mathrm{NiSO} & \left..7 \mathrm{H}_{2} \mathrm{O}\right\} \\ 0,0255 \mathrm{~g} & 0,0259 \mathrm{~g}\end{array}$

3) Angewaudt: 10,0256 $\mathrm{g}$ Kupfer Gefunden: $0,0254 \mathrm{~g}$ Kupfer.

$$
\begin{array}{rrr}
150 \mathrm{~g} \mathrm{NiSO} \mathrm{H}_{4} .7 \mathrm{H}_{2} \mathrm{O} \mid & 0,0254 \mathrm{~g} & , \\
0,0253 \mathrm{~g} &
\end{array}
$$

Das Kupfer wurde in diesen Fällen elektrolytisch bestimmt, nachdem man den durch Hydrosulfit erhaltenen Niederschlag in wenig Salpetersäure gelöst hatte. Die entkupferte Lösung erwies sich in allen Fällen als völlig frei von Nickel. Natürlich bätte der Niederschlag behufs Wägung auch ebenso bequem durch Glüben mit Schwefel im Wasserstoffstrome in Sulfür oder nach Volhard ${ }^{8}$ ) durch Lösen in Salpetersäure und Abdampfen mit Schwefelsäure in wasserfreies Sulfat ubergeführt werden können. Doch wäre dann die Prüung auf Spuren ron Nickel etwas umstäudlicher gewesen.

Die Fällung des Kupfers erfolgt am besten aus schwachsaurer Lösung mit einem möglichst geringen Ueberschusse an Natriumbydrosulfit; andernfalls ist dem Kupfer sehr viel Schwefelkupfer beigemengt. Das Absetzen des Niederschlags wird durch Umrübren und nachträgliches Erhitzen begünstigt.

s) Herr Geheimrath Volhard hatte die Giite, mich brieflich auf diese Methode aufmerksum zu machen. Wie ich in anderen Fällen Gelegenheit hatte, mich zn iberzengen, liefert dieselbe ganz rorzigliche Resultate, die bis an die Grenze der erreichbaren Wägegenauigkeit heraureichen und bietet bei einiger Lebung in der Ausführung wicht die geringsten Schwierigkeiten. 
25. Kobalt.

Kobalt verhält sich gegen Natriumhydrosulfit ganz wie das Nickel. Doch erfolgt die Abscheidung des Sulfids in allen Fällen langsamer wie beim Nickel, besonders in der Kälte. Beim Erwärmen lässt sich aber auch Kobalt quantitativ ausfällen. Wäbrend der Spiegel des Nickelsulfids die Farbe des Metalles besitzt mit einem schwachen Stiche ins Gelbliche, zeigt der Kobaltsulfidspiegel mehr einen Bronzeton.

\section{Palladium.}

Palladiumsalze werden in neutraler wie auch in saurer Lösung durch Natriumbydrosulfit scbon in der Kälte sofort zu Metall reducirt. Der Niederschlag enthält stets etwas Palladiumsulfür. Die Fällung ist eine rollstäudige.

\section{Platin.}

Hydroschweflige Säure wurde von Gröger ${ }^{9}$ ) benützt, um Kaliumplatinchlorid zu Kaliumplatinchlorür zu reduciren. Versetzt man eine Lösung von Wasserstoffplatinchlorid mit Natriumbydrosulfit, so schlägt die gelbe Farbe uugenblicklich in dunkelroth um unter Bildung von Wasserstoffplatinchlorur. Der Farbenumschlag ist auch in verdünnter Lösung sehr augenfällig und kann zum Nachweise des Platins benutzt werden.

Giebt man zu einer Lösung, die im Liter $0,1 \mathrm{~g}$ Platin enthält und nur ganz schwach gelb gefärbt ist, einige Tropfen Natriumhydrosulfitlösung, so wird die Färbung sofort intensiver. Erwärmt man, so scheidet sich Schwefel $a b$, der aber wieder in Lösung geht, während die Flüssigkeit eine rothgelbe Farbe angenommen hat, die in einer mehrere Centimeter dicken Schicht roth erscheint. Die völlig farblose Lösung, welche im Liter 0,01 g Platin enthült, bleibt auch bei Zusatz des Reagens farb1os. Erwärmt man dieselbe aber, bis der ausgeschiedene Schwefel sich wieder gelöst hat, so erhält man eine Flüssigkeit

9) Zeitschr. f. angew. Chem. 1897, Seite 152. 
von schwach gelblicher Farbe, die in $10 \mathrm{~cm}$ dicker Schicht rothgelb erscheint. Eine stark verdünnte Lösung von Natriumhydrosulfit wird beim Erwärmen zwar anfangs auch etwas gelblich gefärbt, aber dann wieder vollkommen farblos.

Auf diese Weise lässt sich Platin leicht neben Gold nachweisen. Die Gold und Platin enthaltende Lösung wird - eventuell nach vorhergegangener Concentration - mit überschlussigem Natriumbydrosulfit versetzt und erwärmt, bis das Gold sich abgesetzt und die über dem Niederschlage stehende Flüssigkeit wieder klar geworden ist. Eine gelbe bis rothe Färbung derselben zeigt die Gegenwart von Platin an.

\section{Selen.}

Auf Lösungen von seleniger Säure wirkt hydroschweflige Säure wie schweflige Säure, nur ungleich energischer. Auch in verdünnteu Iösungen entsteht auf Zusatz von Natriumhydrosulfit bei gewöhnlicher Temperatur sofort ein rother Niederschlag von Selen, während die Reduction von seleniger Säure unter diesen Umstānden nur langsam und unvollständig verläuft.

Selensäure wird durch hydroschweflige Saure ebensowenig reducirt wie durch schweflige Säure, auch nicht beim Erwärmen.

\section{Tellur.}

Tellurige Säure wird auch in sehr starker Verdünnung durch Natriumhydrosulfit augenblicklich zu Tellur reducirt. In einer Lösung, die in Liter $0,1 \mathrm{~g}$ Tellur enthält, bildet sich auf Zusatz eines Tropfens Hydrosulfitlösung sofort eine schwarzbraune Wolke von fein vertheiltem Tellur. Eine Lösung, die im Liter nur $0,01 \mathrm{~g}$ Tellur enthält, nimmt nach Zusatz von Natriumbydrosultit eine lichtbraune Färbung an mit einem Stiche ins Violette. Fubrt man die Reaction in einem Probircylinder aus und sieht von oben durch die Flüssigkeit, so erscheint sie völlig undurchsichtig.

Auch Tellursäure wird bei gewöhvlicher Temperatur $z u$ Tellur reducirt. Giebt man das Reagens zu einer sehr ver- 
dünten Lösung, so fürbt diese sich zuerst violett und scheidet dann schwarzes Tellur ab.

Die Fällung des Tellurs ist vollständig und beim Erwärmen sehr rasch beendigt, während die quantitative Abscheidung des Tellurs durch schweflige Säure bekanntlich längere Zeit erfordert.

Ueberblickt man das Verhalten des Natriumbydrosulfits zu den Salzen der Metalle, so fällt es auf, dass dasselbe nach zwei ganz verschiedenen Richtungen hin reagirt. Einmal tritt es als sehr energisches Reductionsmittel auf, das anderemal führt es die Metalle in Sultide uber. In manchen Fällen wirkt es je nach den Reactionsbedingungen im einen oder im anderen Sinne. Die in der elektrischen Spannungsreihe voranstehenden Metalle werden vorzugsweise als Sulfide gefällt, sofern diese unter den obwaltenden Verhältnissen existenzfăhig sind. Dieso Metallsulfide sind wahrscheinlich erst das Zersetzungsproduct primär gebildeter Hydrosulfite. Dafür spricht der Umstand, dass die Sulfidbildung eine gewisse Zeit beansprucht und dass in manchen Fällen, z. B. beim Zink, Cadmium und Zinn schwer lösliche Hydrosulfite oder Doppelsalze der hydroschwefligen Säure thatsächlich isolirt werden konnten. Die Verwandtschaft der Metalle zum Schwefel ist nicht massgebend für das Verhaiten ihrer Salze zu Natriumhydrosulfit; denu sonst müsste das Kupfer in erster Linie dieser Gruppe zugezählt werden.

Den Salzen der elektropositiveren Metalle gegenuber verhält sich das Natriumbydrosulfit in erster Linie als Reductionsmittel. Ein Ueberschuss desselben bewirkt häufig Sulfidbildung, besonders in der Wärme und bei Gegenwart grösserer Mengen ron freier Süure. Aber auch hier ist vielfach der primäre Vorgang die Reduction zu Metall, das dann durch die hydroschweflige Säure in Sulfid verwandelt wird. Beim Kupfer z. B. lässt sich dies ganz gut verfolgen.

Was nun die Verwendung des Natriumbydrosulfits in der analytischen Chemie betrifft, so stand bisher die unbequeme 
Form und geringe Haltbarkeit des als Paste unter Alkohol aufzubewahrenden Reagens hindernd im Wege. Diesem Uebelstande glaubte ich abhelfen zu können durch Verwendung desselben in Form der beständigen Formaldehydverbindung. Diesbezügliche Versuche zeigten jedoch, dass dieselbe für den gedachten Zweck nicht zu gebrauchen ist, da sie viel zu träge gegenüber den meisten Metallsalzen reagirt. Die Spaltung der Verbindung in der wässrigen Lösung erfolgt nicht rasch genug. Dagegen ist es in jüngster Zeit der Badischen Anilin- und Sodafabrik gelungen, Natriumhydrosulfit in völlig trocknem $\mathrm{Zu}$ stande mit einem Gebalte von $96 \mathrm{pC}$. darzustellen. Dieses Präparat, ein schneeweisses Pulver, das sich in gut verschlossenen Glasbuchsen lange Zeit ohne bemerkbare Zersetzung hält, eignet sich vorzüglich für analytische Zwecke. In dieser Form ist das Reagens einer ausgedehnten Anwendung fähig. Hierüber soll seiner Zeit an anderer Stelle berichtet werden, wenn erst mehr Erfahrungen in der analytischen Praxis gesammelt sein werden.

Ich kann diese Abhandlung nicht schliessen, ohne der Badischen Anilin- und Sodafabrik far die liberale Ueberlassung der verschiedensten Natriumbydrosulfitpräparate meinen wärmsten Dank auszusprechen. 